Z. klin. Chem. u. klin. Biochem.

10. Jg. 1972, S. $108-111$

\title{
Aromatische Carbonsäuren im Urin
}

II. Mitteilung. Eine quantitative Bestimmungsmetbode von (5-Hydroxy-3-indolyl)-essigsäure neben Vanillinmandelsäure aus dem Urin und deren allgemeine Problematik

Von J. Gartzke und E. Majewsiki

Aus dem Medizinisch-Diagnostiscben Institut „Unter den Linden“, Berlin, Leiter: Dr. K. H. Goll

(Eingegangen am 19. August 1971)

(5-Hydroxy-3-indolyl)-essigsäure kann aus dem Urin neben Vanillinmandelsäure nach Extraktion und dünnschichtchromatographischer Abtrennung von den anderen im Urin befindlichen Phenolcarbonsäuren mit sauren Laufmitteln an Kieselgel G mit 2.6-Dichlorchinon-4chlorimid in Gegenwart von Pyridin als Katalysator kolorimetrisch bestimmt werden. Bei der Aufarbeitung des Harnes geht ein größerer Teil der (5-Hydroxy-3-indolyl)-essigsäure durch Zersetzung verloren, so daß die in der Literatur angegebenen Werte nicht den wahren Werten entsprechen.

\section{Aromatic carboxylic acids in urine}

II. A quantitative method for the determination of (5-bydroxy-3-indolyl)-acetic acid and vanillinmandelic acid from urine, and some problems of methodology

Urinary (5-hydroxy-3-indolyl)-acetic acid and vanillinmandelic acid are extracted and separated from the other urinary phenolic carboxylic acids by thin layer chromatography on Kieselgel $G$ with acidic eluants; they can then be determined colorimetrically after reaction with 2.6-dichlorquinone-4-chlorimide with pyridine as a catalyst.

During the extraction of the urine, a large amount of (5-hydroxy-3-indolyl)-acetic acid is lost by decomposition. The literature values for the excretion of this acid are therefore too low.

WVie schon in der I. Mitteilung (1) für 3-Methoxy-4 hydroxy-mandelsäure (Vanillinmandelsäure) beschrieben, ist es nach einer Modifikation der Methode möglich, die (5-Hydroxy-3-indolyl)-essigsäure auf dem gleichen Dünnschichtchromatogramm quantitativ zu bestimmen.

(5-Hydroxy-3-indolyl)-essigsäure ist ein Produkt des Serotoninstoffwechsels. Serotonin wird u. a. in relativ hoher Konzentration in den argyrophilen Zellen der Darmwand gefunden. Bei Tumoren dieser Zellen findet man (5-Hydroxy-3-indolyl)-essigsäure in abnorm großer Menge im Harn. Allerdings haben H. SchIEvelbeIN und Mitarbeiter (2) festgestellt, daß auch z. B. durch Rauchen eine geringe Erhöhung der (5-Hydroxy-3indolyl)-essigsäure-Ausscheidung bewirkt wird. Die im allgemeinen durchgeführten Screening-Tests (3-6) sind einschließlich ihrer üblichen Modifizierungen (3-5) wegen ihrer Unspezifität, Störanfälligkeit, besonders aber wegen der falsch negativen Ergebnisse des öfteren der Kritik unterworfen worden $(3,7)$.

Wir bestimmen die (5-Hydroxy-3-indolyl)-essigsäure in einem Arbeitsgang neben der Vanillinmandelsäure quantitativ nach dünnschichtchromatographischer Auftrennung an Kieselgel $G$, wie von uns schon für die Vanillinmandelsäure beschrieben (Abb. 1, Fleck 4). Auch für die (5-Hydroxy-3-indolyl)-essigsäure hat sich ein 10proz. Pyridinzusatz als Katalysator für die Anfärbung mit GrвBs' Reagenz bewährt. Allerdings findet man die höchste Farbintensität mit Tris-Puffer $\mathrm{pH}^{\circ}$ 7,0 im Gegensatz zur Vanillinmandelsäure, deren höchste
Farbintensität bei pH 9,5 (Citrat-Boratpuffer) liegt. Aus diesem Grunde mußte zur quantitativen Bestimmung von Vanillinmandelsäure und (5-Hydroxy-3-

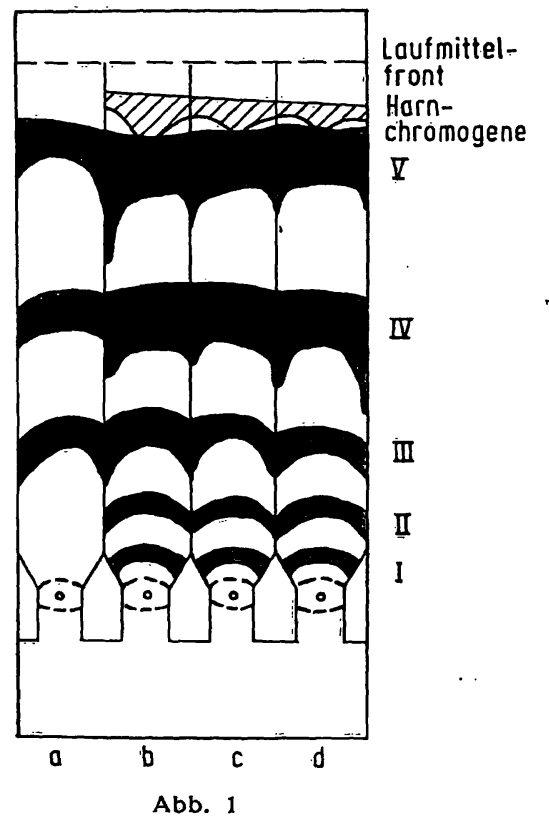

Verteilung der einzelnen Fraktionen auf dem Chromatogramm: I manchmal auftretende nicht identifizierte aromatische Carbon-

II niäure

III Vanillinmandelsäure

V (5-Hydroxy-3-indolyl)-esșigsäure

o-Hydroxy-phenylessigsäure

a: Standards der Substanzen III-V b: Standards der Substanzen III-V plus Harnextrakt
c, d: Harnextrakt 


\section{Optimale \\ Qualltätskontrolle}

im Blut-Gas-Laboratorium

\section{Versatol Acid-Base}

Standard- und Kontrollseren

für $\mathrm{pH}-, \mathrm{CO}_{2}-$ und $\mathrm{pCO}_{2}$-Messungen

in drei Bereichen

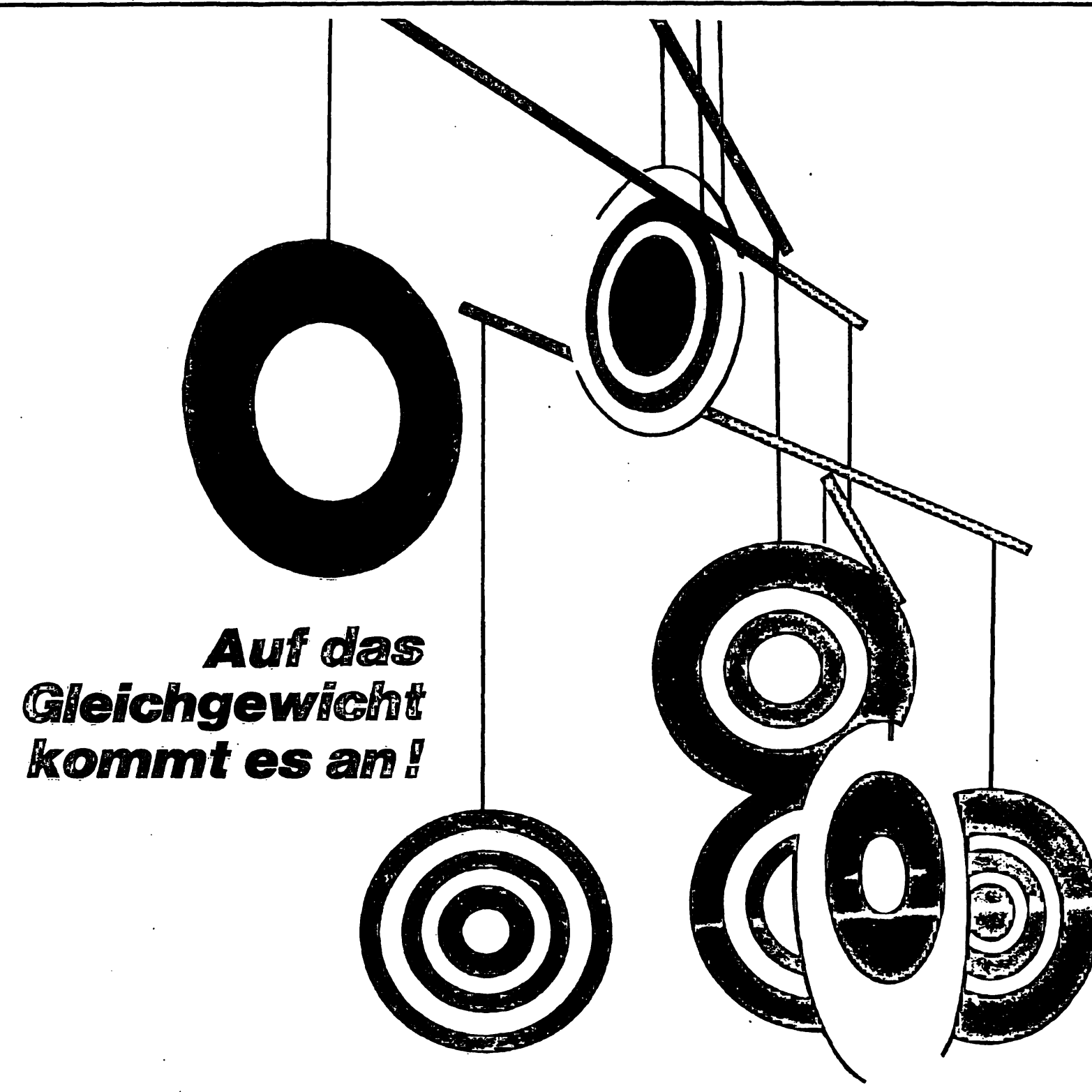

Gödecke Aktiengesellschaft · Berlin 


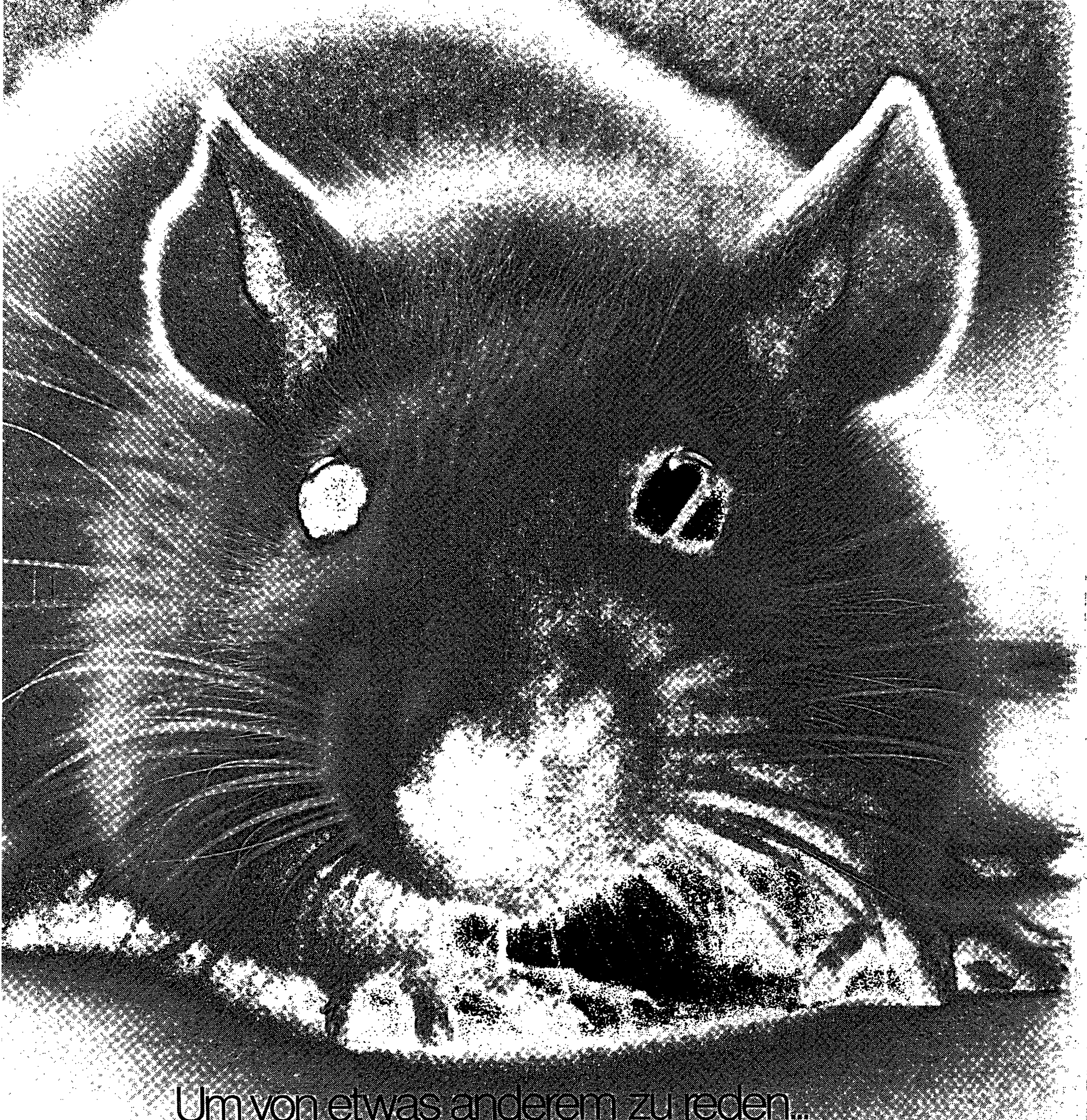

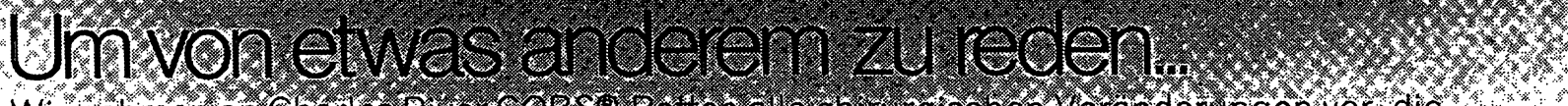

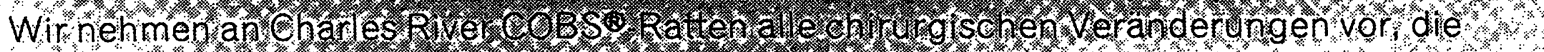

Thren tabohz wecken ens

Wir führen Thy roidektomen auch Thymektomien an neugeborenen Rattenjungen. Wir können jede einzelne dieser verschiedenen chirurgischen veränderungen, oder auch je de beliebige Kombination an jedem von Ihnen bestimmten Tier vornehmen.

Alle Mitglieder des chirurgischen Teams bei Charles River France sind Biologen, die die erforderlichen Eingriffe in schonenden Verfahren so umfassend und vollständig durchżuführen verstehen, daß die Tiere für Versuche optimal geeignet sind.

Wenn Sie chirurgisch veränderte Tiere benötigen, teilen Sie uns bitte thre entsprechenden Wünsche mit.

Was an Charles River Ratten zu machen ist, können Sie uns anvertrauen.

\section{Charles River France fl}


indolyl)-essigsäure nebeneinander die in (1) beschriebene Methode so modifiziert werden, $\mathrm{da} \beta$ eine quantitative Bestimmung aller anfärbbaren Phenolcarbonsäuren möglich ist. Wie aus Abbildung 1 ersichtlich ist, läßt sich weiter dünnschichtchromatographisch auch o-Hydroxyphenyl-essigsäure (Fleck V) nachweisen, ein Abbauprodukt des Phenylalanins, die möglicherweise für die Diagnose einer Alkaptonurie Bedeutung erlangen könnte. Die Bestimmung dieser Phenolcarbonsäure erfordert wieder etwas andere Bedingungen bezüglich ihrer quantitativen Bestimmung. Untersuchungen über eine genauere Identifizierung und Optimierung werden nach weiterer Reinigung der Fraktion später eventuell veröffentlicht.

\section{Ergebnisse und Diskussion}

\section{Extraktion der (5-Hydroxy-3-indolyl)-essigsäure}

Die Unbeständigkeit von 3-Indolylessigsäure gegen Säuren, Basen und Luftsauerstoff ist hinlänglich bekannt.

$\mathrm{Da}$ wir in der Literatur keinen $\mathrm{pK}$-Wert für die NHGruppe der (5-Hydroxy-3-indolyl)-essigsäure oder des Indols fanden, gingen wir mit einem $\mathrm{pH}$-Wert des Harnes von 4 um etwa $2 \mathrm{pH}$-Einheiten unter den pKWert des Essigsäurerestes der 3-Indolyl-essigsäure und verglichen die Ausbeuten mit denen einer stark angesäuerten Probe des gleichen Harnes. Als Extraktionsmittel verglichen wir Äthylacetat und Äther. Die Vollständigkeit der Extraktion konnte wegen der spontanen Decarboxylierung (8) der (5-Hydroxy-3-indolyl)-essigsäure nicht analog der Vanillinmandelsäure durch eine Perforation in der KuTSCHER-STEUDEL-Apparatur überprüft werden. Hier wurde die Vollständigkeit der Extraktion durch quantitative Bestimmung der einzelnen Extrakte einer 6maligen Extraktion von 1 Volumenteil Harn mit jeweils 0,6 Volumenteilen Essigester je Extraktionsschritt ermittelt. Es zeigte sich, daß eine 2malige Extraktion ausreicht, d. h. daß ab der dritten Extraktion dünnschichtchromatographisch keine (5- $\mathrm{Hy}-$ droxy-3-indolyl)-essigsäure mehr nachweisbar ist. Die optimalen Extraktionsbedingungen sind also die gleichen wie bei der Vanillinmandelsäure (stark angesäuerter Harn, Äthylacetat als Extraktionsmittel). Eine Vorbehandlung des Harnes mit Florisil, wie sie für Vanillinmandelsäure möglich ist (10), führt bei der (5-Hydroxy3-indolyl)-essigsäure zu erheblichen Verlusten.

\section{Dïnnscbicbtchromatograpbie, Elution und quantitative Be- stimmung}

Die Dünnschichtchromatographie erfolgt genau wie für Vanillinmandelsäure beschrieben an Kieselgel $G$ mit Eisessig/Benzol/Methanol $=4+45+8(\mathrm{v} / \mathrm{v})$ als mobile Phase, jedoch witd nach Entwicklung der Platte zum Abstumpfen der Essigsäure nicht mit Boratpuffer $\mathrm{pH} 9,5$ besprüht, sondern lediglich in eine mit $\mathrm{NH}_{3}$ gesättigte Kammer gestellt. Nach Besprühen mit GIBBs' Reagenz und erneutem Einstellen in die „, $\mathrm{NH}_{3}-\mathrm{Kammer}$ “" wird die Vanillinmandelsāure wie üblich mit Borat- puffer $\mathrm{pH} 9,5$ plus $10 \%$ Pyridin und die (5-Hydroxy-3indoly1)-essigsäure mit Tris-Maleinsäure-Puffer $\mathrm{pH} 7,0$ plus $10 \%$ Pyridin eluiert. Die (5-Hydroxy-3-indolyl)essigsäure wird nach 20-25 Min. bei $580 \mathrm{~nm}$ im Absorptionsmaximum der reinen (5-Hydroxyr-3-indolyl)essigsäure kolorimetriert. Das Maximum ist bei der „Harn-Hydroxyindolessigsäure" etwas in das längerwellige Gebiet verschoben, doch hebt sich das Maximum nur wenig aus einem breiten Plateau heraus, so daß die Verschiebung für diese Zwecke vernachlässigt werden kann. Wesentlich problematischer als bei der stabilen Vanillinmandelsäure ist die Instabilität des Indolringes sowie des Essigsäurerestes. Das macht sich - wie Abbildung 2 zeigt - bei den "Chromatographieverlusten" (in Abb. 2 die Differenz zwischen Kurve 1 und 2) be-

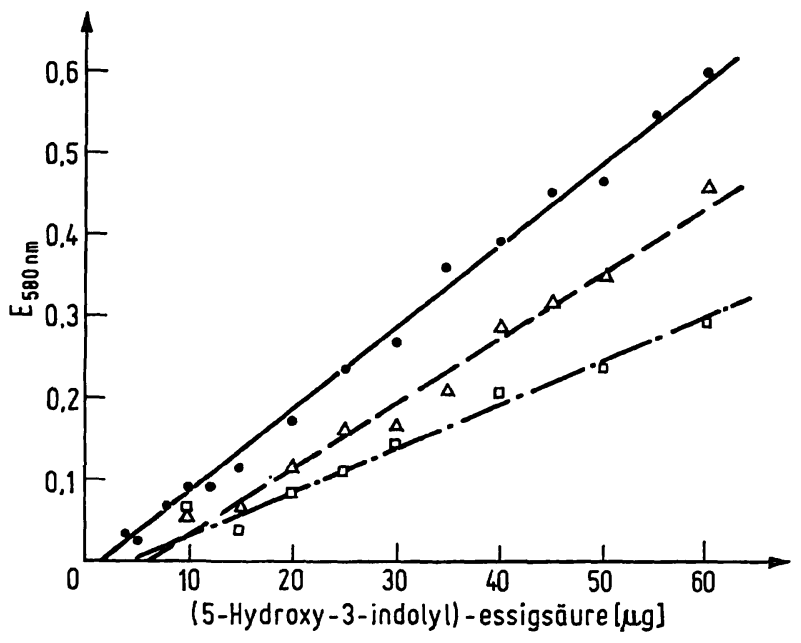

Abb. 2

Eichkurven für (5-Hydroxy-3-indolyl)-essigsäure reine Hydroxyindolessigsäure ohne sowie nach Elution ohne Dünnschichtchromatographie

reine Hydroxyindolessigsäure nach Dünnschichtchromatographie

- - Hydroxyindolessigsäure plus Harnextrakt nach Dünnschichtchromatographie und Elution gemessen gegen Harnextrak (Spekol $580 \mathrm{~nm}$ )

merkbar. "Chromatographieverluste" durch Zersetzung bei der Reinigung an hochaktiven Sorbentien und unvollständige Elution sind aus der Alkaloidchemie bei Indolalkaloiden hinlänglich bekannt. SAGI (9) schlägt wegen unvollständiger Elution Celluloseschichten vor. Wir fanden bei Verwendung von Celluloseschichten mit Differenzen von etwa $\pm 3 \%$ keine Signifikanz. Um alle Verluste abschätzen zu können, extrahierten und chromatographierten wir reine (5-Hydroxy-3-indolyl)-essigsäure nach 24stdg. Stehen, wobei im Gegensatz zu Harn, bei dem der (5-Hydroxy-3-indolyl)-essigsäure-Gehalt auch nach Ansäuern durch Stehen im Kühlschrank ständig abnimmt, kein Verlust nachgewiesen werden konnte. Erst nach der 4maligen Extraktion (1) betrug der Verlust an (5-Hydroxy-3-indolyl)-essigsäure 17 bis $26 \%$. Durch die Dünnschichtchromatographie kamen weitere $6-12 \%$ Verlust hinzu. Verluste durch Dünnschichtchromatographie sind weniger problematisch, da der Gehalt an (5-Hydroxy-3-indolyl)-essigsäure mittels eines mitgelaufenen Arbeitsstandards bestimmt wird. 
Einige Autoren $(3,11,12)$ haben - auch bei der allgemein üblichen Methode nach UDENFRIEND - Verluste gefunden, sie aber nicht als Folge einer möglichen Zersetzung interpretiert.

Andere Autoren $(13,14)$ versuchten deshalb ${ }^{-}$u. a. die dünnschichtchromatographische Trennung der aromatischen Carbonsäuren des Urins nach ihrer Reaktion mit diazotiertem $p$-Nitranilin, doch wird hierdurch die Stabilität der Carbonsäuren nicht erhöht, sondern es treten höchstwahrscheinlich zusätzliche Verluste durch die Instabilität des Farbstoffes auf. Dies ist sicher auch der Grund, warum sich die recht elegante Methode bisher nicht durchgesetzt hat.

\section{Material und Methoden}

1. konz. Salzsäure p. a.

2. $\mathrm{NaCl}$ p. a.

3. Äthylacetat p. a.

4. Natriumsulfat sicc. $\mathrm{DAB} 7$

5. 60proz. Alkohol

6. Kieselgel G (Merck)

7. Benzol (zur Molekulargewichtsbestimmung)

8. Methanol p. a.

9. Eisessig p. a.

10. Tris-Maleinsäure-Puffer $\mathrm{pH} 7$ $6,05 \mathrm{~g}$ Tris(hydroxymethyl)-aminomethan und 5,8 $\mathrm{g}$ Maleinsäure werden in $250 \mathrm{ml}$ Wasser gelöst, mit $48 \mathrm{ml} \mathrm{N} \mathrm{NaOH}$ versetzt und mit dest. Wasser auf 11 aufgefüllt (15)

11. Pyridin reinst (frisch dest.)

12. 2.6-Dichlor-chinon-4-chlorimid reinst

13. Sprühreagenz (0,1 proz.)

2.6-Dichlor-chinon-4-chlorimid in Alkohol

Aufbewahrung im Kühlschrank!

14. (5-Hydroxy-3-indolyl)-essigsäure-Standard ( $1 \mathrm{mg} / \mathrm{ml}$ (5-Hydroxy-3-indolyl)-essigsäure in aldehydfreiem Alkohol) Im Kühlschrank etwa 2 Wochen haltbar!

\section{Arbeitsvorschrift}

$50 \mathrm{ml}$ 24-Stdn-Sammelharn oder entsprechend weniger werden mit konz. $\mathrm{HCl}$ auf $\mathrm{pH} 1$ gebracht und mit Natriumchlorid gesättigt. Anschließend wird der Harn $2 \mathrm{mal}$ (bei gleichzeitiger Bestimmung von Vanillinmandelsäure $3 \mathrm{mal}$ ) mit je $30 \mathrm{ml}$ Essigester extrahiert (bei Emulsionsbildung ist zur Phasentrennung zu zentrifugieren). Die vereinigten Essigesterextrakte werden über Natriumsulfat getrocknet und der Essigester anschließend im Vakuum abgedampft. Nach Lösen in 60proz. Äthanol wird wie für Vanillinmandelsäure (1) beschrieben dünnschichtchromatographiert. Es werden im Gegensatz zur Vanillinmandelsäure $20 \mu \mathrm{g}$ (5-Hydroxy-3-indolyl)-essigsäure als Standard aufgetragen. In Abänderung der Vanillinmandelsäure-Vorschrift wird auf ein Besprühen der Platte mit Boratpuffer pH 9,5 verzichtet und nach dem Einstellen in eine mit Ammoniak gesättigte Kammer gleich mit dem Sprühreagenz besprüht. Dann wird die Platte zur Farbvertiefung wiederum einige Sekunden einer $\mathrm{NH}_{3}$-Atmosphäre ausgesetzt, die (5-Hydroxy-3-indolyl)-essigsäure- bzw. die Vanillinmandelsäure-Bande abgekratzt und mit 5,4 ml Trispuffer pH 7,0 (bei Vanillinmandelsäure entsprechend mit Boratpuffer $\mathrm{pH}$ 9,5), 0,6 ml Pyridin und 0,1 ml Sprühreagenz eluiert. Es wird nach Schütteln $10 \mathrm{Min}$. bei $3500 \mathrm{U} / \mathrm{Min}$. zentrifugiert, die überstehende Lösung dekantiert und die (5-Hydroxy-3-indolyl)essigsäure $20 \mathrm{Min}$. nach Beginn der Elution bei $580 \mathrm{~nm}$ (die Vanillinmandelsäure 25 Min. nach Elutionsbeginn bei $620 \mathrm{~nm}$ ) und einer Schichtdicke $\mathrm{d}=1 \mathrm{~cm}$ gegen einen Blindwert gemessen, der der Leitbahn des Standardwertes entnommen wurde (Vanillinmandelsäure und (5-Hydroxy-3-indolyl)-essigsäure-Standard be- nötigen nur eine Leitbahn, aber zwei Blindwerte). Wegen der "Chromatographieverluste" und anderer möglicher Fehlerquellen wird die Berechnung der ,Harn-Hydroxyindolessigsäure“-Konzentration mit dem Standard empfohlen. Die Empfindlichkeit der Methode kann durch Auftragen größerer Mengen des Harnextraktes (wir tragen Extrakt, der etwa $3 \mathrm{ml}$ Harn entspricht, auf), Verringerung des Volumens der Elutionsflüssigkeit oder größere Schichtdicke noch gesteigett werden.

\section{Normalwerte}

Aufgrund unserer Ergebnisse spiegeln die in der Literatur angegebenen Normalwerte der (5-Hydroxy-3-indolyl)-essigsäure-Ausscheidung nicht den wahren Hydroxyindolessigsäuregehalt des Harnes wider. Wenn auch aus den Eichkurven der Abbildung 2, die Mittelwerte großer Serien darstellen, ein zu großer Verlust durch die Dünnschichtchromatographie hervorgeht (vgl. in dieser Mitt. angegebene Werte für reine (5-Hydroxy-3-indolyl)essigsäure), so ist es doch offensichtlich, daß die Verluste von (5-Hydroxy-3-indolyl)-essigsäure des Harnes größer sind als bei reiner Substanz. Sie hängen in beiden Fällen zusätzlich von der Konzentration ab. Die günstigsten Konzentrationen liegen nach Dünnschichtchromatographie für die kolorimetrische Bestimmung zwischen $40-50 \mu \mathrm{g}$ (5-Hydroxy-3-indolyl)-essigsäure pro Fleck. Oberhalb $60 \mu \mathrm{g}$ beginnen die Werte für die Eichgeraden sehr stark zu streuen. Zusätzliche Fehler gelangen durch Zersetzung der „Harn-Hydroxyindolessigsäure“" bereits nach 24stdg. Stehen des Harnes im Kühlschrank selbst nach Ansäuern in das Ergebnis. Ob diese Zersetzung bakteriell $(16,17,18)$ oder enzymbedingt ist oder andere Ursachen hat, konnte nicht ermittelt werden. Das könnte aber durch Isolierung möglicherweise im Harn vorkommender Indolessigsäureoxydase sowie anderer Oxydasen ermittelt werden $(17,19)$.

Die gesamten Verluste sind in ihrer Größe nur schwer oder gar nicht reproduzierbar und werden für „HarnHydroxy-indolessigsäure" mit $40-60 \%$ abgeschätzt. Dies befindet sich auch etwa in dem Bereich wie er für Isolierung von 3-Indolylessigsäure aus Pflanzenextrakten angegeben worden ist (20). Die Wiederfindungsraten für zum Harn zugegebene reine (5-Hydroxy-3-indolyl)essigsäure lagen zwischen 65 und $73 \%$. Nach Literaturangaben modifizierte Normalwerte betragen 1-9 mg (5-Hydroxy-3-indolyl)-essigsäure/24-Stdn.-Harn, und bei Rauchern 14,85 mg/24 Stdn.-Harn (21).

Wir fanden bei unserer Methode einen Mittelwert von $5,17 \mathrm{mg} / 24-S t d n .-H a r n$ mit einem s-Bereich von $\pm 4,22$ $\mathrm{mg}$. Berechnet man auf das übliche $\mathrm{Maß}$ der Streuung von $2,3 \mathrm{~s}$, so erhält man $\pm 9,69 \mathrm{mg}$. Dies zunächst unsinnig erscheinende Ergebnis gewinnt unter Beachtung folgender Gesichtspunkte an Realität:

1. Der Gehalt an (5-Hydroxy-3-indolyl)-essigsäure ist wegen der schnellen Zersetzung beim Stehen und bei der Aufarbeitung nicht exakt zu ermitteln.

2. Aus diesem Grunde ist die in der Literatur beschriebene biologische Streuung seht hoch.

3. Das Alter des Harns ist für die Streuung mitverantwortlich. 
4. Die für die Mittelwertberechnung verwendeten Harne konnten nicht nach Rauchern und Nichtrauchern selektiert werden.

Unter Berücksichtigung dieser Punkte ist ein wahrer Normalwert von etwa $25 \mathrm{mg} / 24 \mathrm{Stdn}$-Harn zu erwarten, der aber in der Diagnostik kaum praktische Bedeutung haben dürfte, zumal alle Bestimmungsmethoden mit ähnlich großen Verlusten arbeiten. Der Normalwert sollte daher anhand von Erfahrungen für jede Methode gesondert ermittelt werden.

\section{Literatur}

1. GARTZKE, J. und E. MAJEWSKr, diese Z. 9, 18 (1971). - 2. Schievelbein, H., U. Surberg und E. WerLe, Klin. W/schr. 40, 52 (1962). - 3. Schön, H., W. Ordnung und E. Scrumd, Klin. Wschr. 38, 405 (1960). - 4. Henderson, B. M., D. J. Campbell und B. J. Spronle, Canad. med. Ass. J. 84, 1238 (1961). - 5. KIRBERGER, E., Dtsch. med. Wschr. 91, 2128 (1966). - 6. Mustala, O. O., J. J. Tuomisto und M. M. Airaksimen, Scand. J. Clin. Laborat. Invest. 16, 655 (1964). - 7. Schievelaern, H., Dtsch. med. Wschr. 93, 2134 (1968). - 8. Deverall, B. J., Nature (London) 207, 828 (1965). - 9. SAGI, F., Bot. Kozlem 55, 107 (1968). - 10. WeILMalherbe, H., diese Z. 2, 161 (1964). - 11. Carcasona, A., J. C. Cervos-Navarro und F. Unterharvscheidt, Klin. Wschr.
37, 253 (1959). - 12. Schmid, E., L. Zicha, J. Krautheim und J. Blumberg, Med. exper. 7, 8 (1962). - 13. Ilinow, P. und R. PanschewA, 1. Nationaler Kongreß für Klin. Labordiagnostik Sofia 12.-14. 11. 1970. - 14. v. SrudNitz, W., Klin. Wschr. 45, 307 (1967). - 15. Gomori, G., Proc. Soc. exper. Biol. Med., N. Y. 68, 354 (1948). - 16. StrzelczyK, E. und J. M. Karwowska, Pol. J. Soil Sci 2, 59 (1969). - 17. Gaspar, Th., Compt. Rend. Acad. Sc. Serv. D 271, 928 (1970). - 18. PArisir, W. R., Z. Pflanzenphysiol. 60, 296 (1969). - 19. Mato, M. C. und J. Mendez, Geoderma 3, 255 (1970). - 20. MANN, J. D. und E. G. JAwORSKI, Planta 92, 285 (1970). - 21. GolL, K. H., Ärztekalender der DDR 1971 , Seite 431 .

Dipl.-Chem. J. Gartzke und E. Majewrki DDR-108 Berlin, Unter den Linden 40 